\title{
JC Virus Infection
}

National Cancer Institute

\section{Source}

National Cancer Institute. IC Virus Infection. NCI Thesaurus. Code C128830.

An infection that is caused by JC virus. 